\title{
Autonomic control of asystolic vasovagal syncope
}

\author{
David L Jardine, Hamid Ikram, Ian G Crozier
}

\begin{abstract}
A 30 year old woman with a lifelong history of severe, recurrent, vasovagal syncope became asystolic for 30 seconds after 37 minutes of $60^{\circ}$ head-up tilt. During early tilt, sympathetic activity, heart rate, left ventricular contractility, and cardiac output increased. Mean blood pressure was initially maintained. Presyncope was associated with maximal contractility and bradycardia despite sustained sympathetic activity. Subsequently, asystole occurred associated with complete withdrawal of muscle nerve sympathetic activity. In asystolic vasovagal reactions, presyncope may be triggered by increased left ventricular contractility and is associated with increased levels of parasympathetic and sympathetic activity. Asystole and peripheral vasodilatation may be caused by sudden and complete withdrawal of the increased sympathetic activity.
\end{abstract}

(Heart 1996;75:528-530)

Keywords: head-up tilt; vasovagal syncope; asystole

Some patients with severe, recurrent, vasovagal syncope have been shown to become asystolic during tilt-table testing. ${ }^{12}$ Their syncopal episodes may be rapid in onset and prolonged, sometimes causing injury. It is generally believed that this condition does not carry an increased mortality despite occasional severe episodes which mimic sudden cardiac death. ${ }^{1}$ The term "malignant vasovagal syncope" has been used to convey the significant morbidity experienced by some patients. ${ }^{2}$

We present a patient with lifelong recurrent syncope and describe detailed haemodynamic and autonomic assessment during an episode of tilt-induced asystole. To our knowledge, this is the first microneurographic record of vasovagal asystolic syncope. Previous reports on similar patients have not included muscle sympathetic activity or heart rate variability. ${ }^{1-3}$ Others have recorded either heart rate variability $^{4-6}$ or muscle sympathetic activity ${ }^{7-9}$ during vasovagal syncope but not simultaneously, and not during asystole in a patient with a "malig- nant history". We also report the associated haemodynamic changes in an attempt to demonstrate how the autonomic nervous system controls the haemodynamics of an asystolic vasovagal reaction.

\section{Case report}

A 30 year old woman was referred for investigation after a postoperative cardiovascular collapse. She awoke on the recovery ward shortly after a general anaesthetic for a vaginal hysterectomy and suddenly lost consciousness while still in a horizontal position. She was found to be hypotensive with a pulse rate of $36 / \mathrm{min}$. One minute later she became pulseless and apnoeic. Cardiopulmonary resuscitation was initiated and she recovered within 3 minutes. Investigations after recovery showed no evidence of perioperative myocardial ischaemia or haemorrhage. She gave a lifelong history of fainting, her previous episodes being precipitated by illness, intense excitement, heat, cold, or venepuncture. On examination there was no evidence of postural hypotension, carotid hypersensitivity, or cardiac disease. Her electrocardiogram, electrophysiological studies, 24 hour Holter tape, and echocardiogram were normal.

\section{Methods}

The patient underwent $60^{\circ}$ head-up tilt table testing to confirm the suspected diagnosis of malignant vasovagal syncope. After a light, caffeine-free lunch she was positioned horizontally and supine on the tilt table with foot support. Cutaneous electrodes for thoracic impedance and the electrocardiogram (ECG) were placed. Microneurography needles were inserted in the right leg to record post ganglionic sympathetic activity from the superficial peroneal nerve. ${ }^{10}$ The following variables were recorded continuously on computer for 15 minutes before tilt, 37 minutes during tilt until syncope, and 15 minutes after tilt during recovery: heart rate (HR) from the ECG; low frequency $(0.06-0.1 \mathrm{~Hz})$ heart rate variability (LFHRV) from a computer-stored tachogram using 256 beat samples for spectral analysis of sympathetic activity; ${ }^{6}$ intra-arterial systolic and diastolic blood pressure; total thoracic impedance and its first time differential from the 

recordings of intra-arterial blood pressure (BP), muscle nerve sympathetic activity (MSA), electrocardiogram (ECG), and thoracic

Sympathetic activity displayed in mean voltage neurogram (time constant $0 \cdot 1 \mathrm{~s}$ ). Paper speed 5 $\mathrm{mm} / \mathrm{s}$.
Figure 1 Simultaneous impedance $(d Z / d t)$
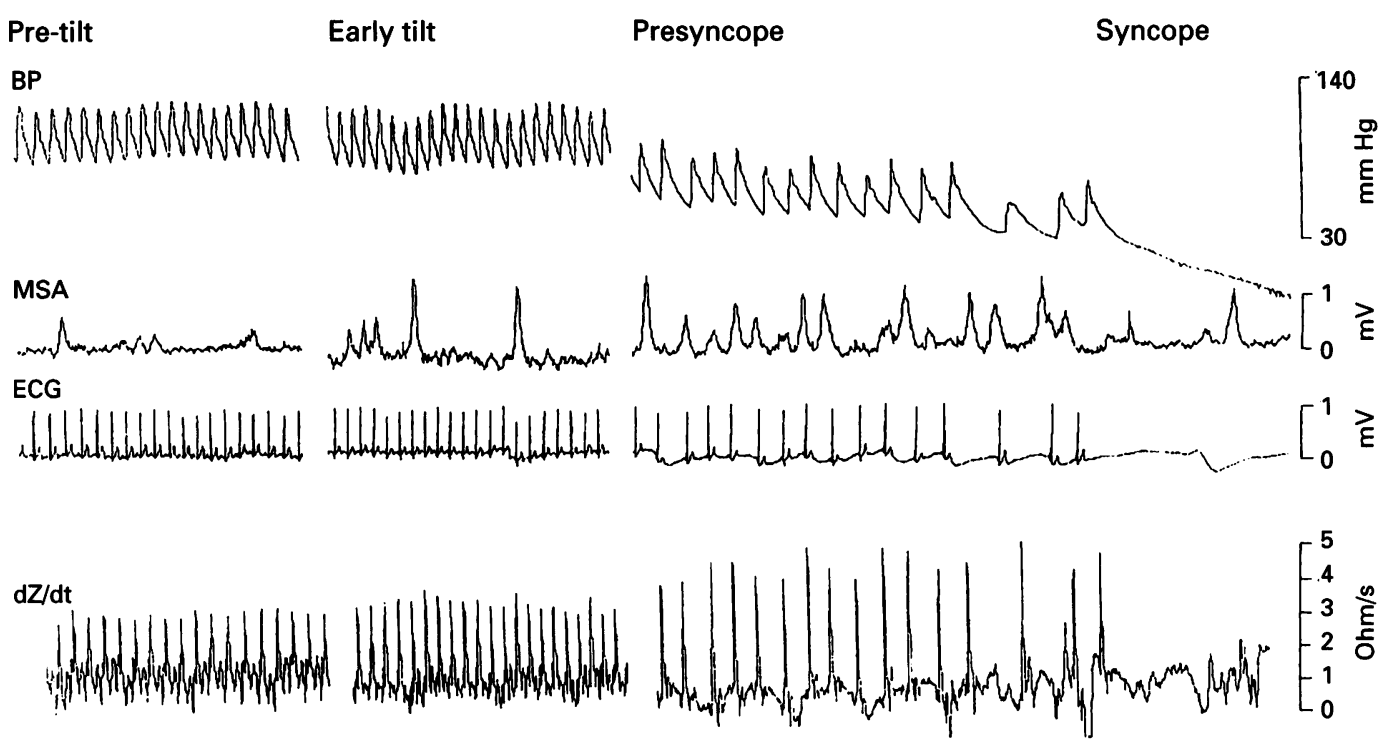

Minnesota impedance cardiograph model $304 \mathrm{~B},{ }^{11}$ and muscle sympathetic activity (MSA) from the integrated voltage neurogram using burst frequency. Bursts of sympathetic activity were recognised by their characteristic morphology and their relation to ECG and blood pressure. ${ }^{10}$

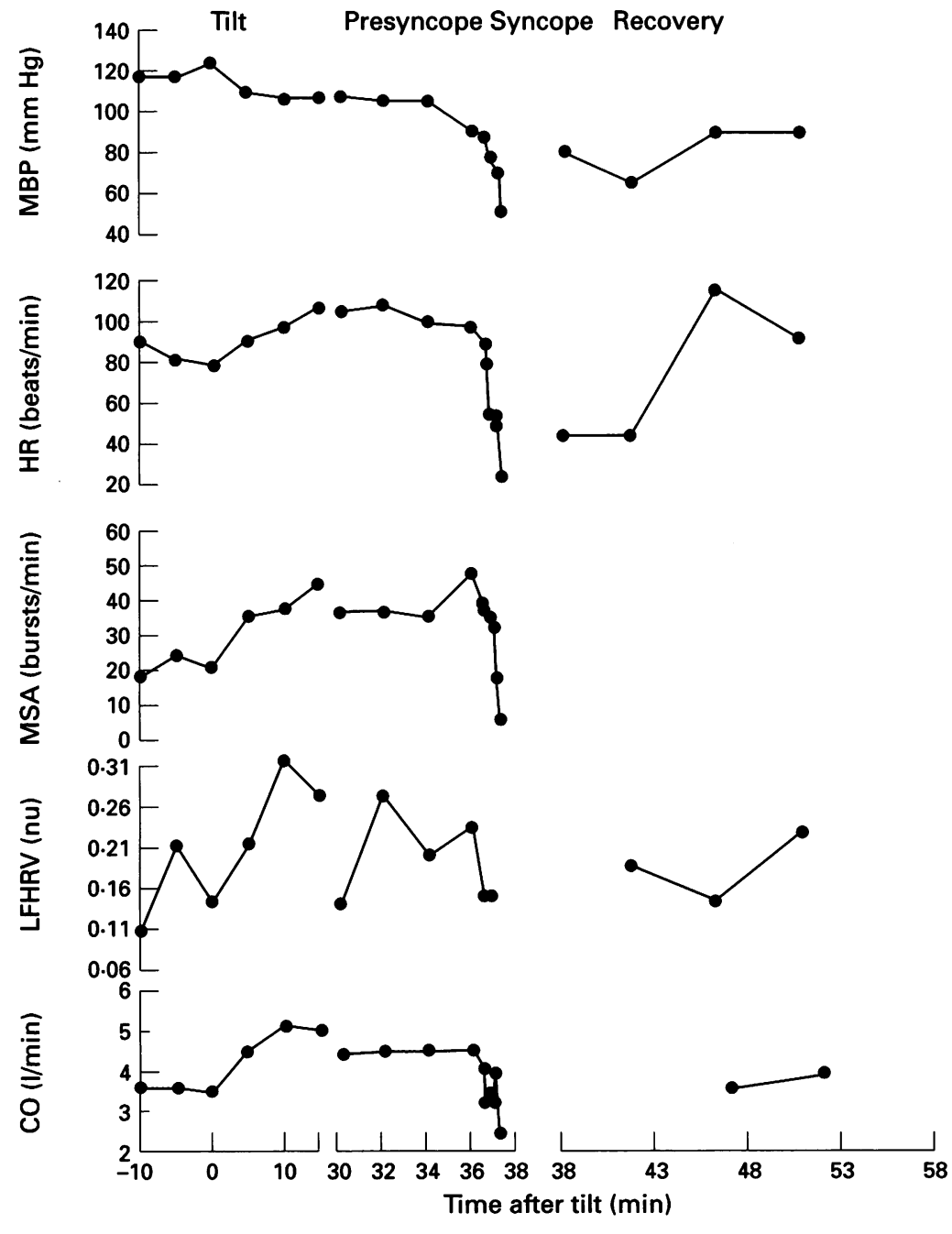

Figure 2 The relation between mean blood pressure (MBP), heart rate (HR), muscle nerve sympathetic activity (MSA), low frequency heart rate variability (LFHRV), and cardiac output $(C O)$ during $60^{\circ}$ head-up body tilt and syncope. Time refers to minutes after tilting. The muscle sympathetic nerve recording field was lost 20 seconds after the onset of syncope.
Mean blood pressure (MBP) was derived from systolic and diastolic blood pressure. Stroke volume was derived from thoracic impedance using the Kubicek equation. ${ }^{11}$ Cardiac output (CO) was derived from stroke volume and $H R$. Left ventricular contractility was estimated using the Heather index (HI), derived from thoracic impedance and the systolic ejection time. ${ }^{11}$

\section{Results}

Initially, when the patient was in the horizontal position, all haemodynamic variables were within normal limits for our laboratory. Immediately after tilt there was an increase in MSA, LFHRV, HI, and HR (figs 1 and 2). During tilt, MBP and CO were maintained for 30 minutes until $\mathrm{HI}$ became maximal and the patient began to feel unwell. At 30 minutes, presyncope began and $H R$ and MBP began to fall despite sustained MSA, LFHRV, and CO. At 36 minutes, MSA surged and then rapidly fell over 60 seconds. Asystole developed lasting for 30 seconds and the patient became syncopal. She was immediately tilted back to the horizontal and the microneurographic recording field was lost during recovery.

\section{Discussion}

The vasovagal response is defined as the development of arteriolar dilatation and inappropriate bradycardia leading to hypotension and loss of consciousness. ${ }^{12}$ The vasodilatation is thought to be due to sympathetic withdrawal and the bradycardia to simultaneous parasympathetic activation, although in humans the exact sequence and the relative importance of these mechanisms remains unclear. ${ }^{1314}$ The response may be triggered in the brainstem by a from the heart or by emotional stress in the cortico-hypothalamic centres of the brain. In some patients the resulting transient autonomic imbalance is severe and reversible asystole may develop in the absence of sinus node disease or carotid hypersensitivity. ${ }^{1-3} \mathrm{We}$ recorded autonomic activity continuously durparadoxical increase in baroreceptor firing rate 
ing an episode of haemodynamically monitored vasovagal asystole, in an attempt to determine the control mechanisms responsible.

The onset of presyncope coincided with the peak level of left ventricular contractility. This lends support to a proposed trigger mechanism for vasovagal syncope, namely the paradoxical stimulation of the central low pressure baroreceptors when left ventricular contractility increases in the setting of a reduced central volume. ${ }^{1516}$ However, recent experiments on anaesthetised dogs did not demonstrate this reflex $^{17}$ and vasovagal syncope has been induced in cardiac transplant recipients by head-up tilting. ${ }^{18}$ Some of these patients had no evidence of cardiac reinnervation which argues against the importance of a cardiac reflex in vasovagal reactions.

Presyncope was associated with a gradual fall in heart rate, blood pressure, and total peripheral resistance despite sustained sympathetic activity. The inappropriate bradycardia can only be explained by an increase in parasympathetic activity occurring at the same time. Therefore both components of the autonomic nervous system were activated simultaneously, rather than sequentially as hypothesised in the diphasic model of vasovagal syncope. ${ }^{19}$ This has not been previously demonstrated and contrasts with other reports of vasovagal reactions which show tachycardia in association with increased MSA during presyncope. ${ }^{7-9}$ The progressive fall in blood pressure despite increased MSA may have been due to an active cholinergic vasodilatory mechanism or the release of adrenaline..$^{2021}$ Alternatively the splanchnic circulation, under separate sympathetic control, may have been relatively more vasodilated at this time. ${ }^{22} 23$ When sympathetic withdrawal did occur, asystole and severe vasodilation were observed, resulting in prolonged syncope. This is consistent with previous demonstrations of vasodilatation triggered by a sudden fall in MSA suggesting a passive mechanism..$^{7-9}$ Similarly, the onset of asystole was related to the sudden withdrawal of sympathetic activity, which allowed the unopposed parasympathetic activity to dominate. The reciprocal nature of the autonomic balance between parasympathetic and sympathetic action was therefore restored.

In this study we have attempted to document as accurately as possible the autonomic changes during tilt-induced asystolic syncope. In the intact human it is not possible directly to measure parasympathetic activity or global sympathetic activity but MSA and low frequency heart rate variability currently provide the best dynamic measures of sympathetic activity. Unfortunately, neither forearm (muscle) nor splanchnic blood flow were measured. Stroke volume and left ventricular contractility were measured indirectly via impedance cardiography, a technique that has been validated for detecting rapid changes in these variables. ${ }^{11}$

In summary, we observed the autonomic and haemodynamic changes that accompanied tilt-induced asystolic vasovagal syncope. We found there was an appropriate increase in sympathetic activity in response to tilt, with cardiac output and blood pressure initially being maintained. The bradycardia and hypotension of presyncope were associated with maximal left ventricular contractility and were due to increased parasympathetic activity occurring on a background of increased sympathetic activity. The usual reciprocal relation between parasympathetic and sympathetic activity was therefore disturbed and the result was prolonged asystole associated with sudden withdrawal of sympathetic activity. This loss of reciprocity, preceding an episode of asystole has not been previously demonstrated but has been implicated as a mechanism for sudden cardiac death..$^{24}$

1 Milstein S, Buetikofer J, Lesser J, Goldenberg IF, Benditt DG, Gornick C, et al. Cardiac asystole: A manifestation of neurally mediated hypotension-bradycardia. $₹ \mathrm{Am}$ Coll Cardiol 1989;14:1626-32.

2 Maloney JD, Jaeger FJ, Fouad-Tarazi FM, Morris HH. Malignant vasovagal syncope: prolonged asystole provoked by head-up tilt. Cleve Clin 7 Med 1988;55:542-8.

3 Goldstein DS, Spanarkel M, Pitterman A, Toltzis R, Gratz E, Epstein S, et al. Circulatory control mechanisms in vasodepressor syncope. Am Heart $₹$ 1982;104:1071-5.

4 Baharav A, Mimouni M, Lehrman-Sagie, Izraeli S, Akselrod S. Spectral analysis of heart rate in vasovagal syncope: the autonomic nervous system in vasovagal syncope. Clin Auton Res 1993;3:261-9.

5 Lepicovska V, Novak P, Nadeau R. Time-frequency dynamics in neurally mediated syncope. Clin Auton Res 1992;2:317-26.

6 Lipsitz LA, Mietus J, Moody GB, Goldberger AL. Spectral characteristics of heart rate variability before and during postural tilt. Circulation 1990;81:1803-10.

7 Wallin BG, Sundlof G. Sympathetic outflow to muscles during vasovagal syncope. F Auton Nerv Syst 1982;6: $287-91$.

8 Saunders JS, Fergusson DW. Profound sympathoinhibition complicating hypovolaemia in humans. Ann Intern Med 1989;111:439-41.

9 Yatomi A, Iguchi A, Uemura K, Sakamoto N, Iwase S, Mano T. A rare case of recurrent vasodepressive attacks of 2-hours duration: Analysis of the mechanism by muscle sympathetic nerve activity recording. Clin Cardiol 1989; 12:164-8.

10 Sunlof G, Wallin BG. Human muscle nerve activity at rest. Relationship to blood pressure and age. $f$ Physiol 1978;274:621-37.

11 Mohapatra S. Non invasive cardiovascular monitoring by electrical impedance technique. London: Pitman Medical, 1981:70-172.

12 van Lieshout J, Wieling W, Karemaker JM, Eckberg DL. The vasovagal response. Clin Sci 1991;81:575-86.

13 Kosinski DJ, Grubb BP, Temesy-Armos P. Pathophysiological aspects of neurocardiogenic syncope: current conlogical aspects of neurocardiogenic syncope: current
cepts and new perspectives. $P A C E$ 1995;18:716-24.

14 Rea RF, Thames MD. Neural control mechanisms and vasovagal syncope. $\dot{f}$ Cardiovasc Electrophysiol 1993;4: v8sovagal

15 Shalev Y, Gal R, Tchou PJ, Anderson AJ, Avitall B, Akhtar $\mathrm{M}$, et al. Echocardiographic demonstration of decreased left ventricular dimensions and vigorous myocardial contraction during syncope induced by head-up tilt. $\mathcal{F} \mathrm{Am}$ Coll Cardiol 1991;18:746-51.

16 Thoren P. Role of cardiac vagal C-fibres in cardiovascular control. Rev Physiol Biochem Pharmacol 1979;86:1-94.

17 Al-Timman JKA, Hainsworth R. Reflex vascular responses to changes in left ventricular pressures, heart rate and inotropic state in dogs. Exp Physiol 1992;77:455-69.

18 Fitzpatrick AP, Banner N, Cheng A, Yacoub M, Sutton R. Vasovagal reactions may occur after orthotopic heart transplantation. $\mathcal{F}$ Am Coll Cardiol 1993;21:1132-7. 19 Graham DT, Kabler JD, Lunsford LL. Vasovagal fainting: a

20 Saunders JS, Mark AL, Fergusson DW. Evidence for cholinergically mediated vasodilation at the beginning of exercise in humans. Circulation 1989;79:815-24.

21 Sander-Jensen K, Secher NH, Astrup A, Christensen NJ, Giese J, Schwartz TW, et al. Hypotension induced by Physiol 1986;251:R742-8.

22 Abboud FM, Eckberg DL, Johannsen UJ, Mark AL Carotid and cardiopulmonary baroreceptor control of Carotid and cardiopulmonary baroreceptor control of splanchnic and forearm vascular resistance

23 Johnson JM, Rowell LB, Niederberger M, Eisman MM. Human splanchnic and forearm vasoconstrictor responses to reductions of right
sures. Circ Res 1974;34:515-24.

24 Engel GL. Psychological stress, vasodepressor (vasovagal) syncope, and sudden death. Ann Intern Med 1978;89: 403-12. 